Benchmarks

\title{
ISH-guided freeze-matrix assisted punches: technique for extracting punches from thin slide-mounted tissues for DNA methylation analysis
}

\section{Lawrence S. Own and Paresh D. Patel \\ Molecular and Behavioral Neuroscience Institute, Department of Psychiatry, and Neuroscience Graduate Program, University of Michigan, Ann Arbor, MI, USA}

BioTechniques 53:175-180 (September 2012) doi 10.2144/0000113912

Keywords: DNA methylation; tissue extraction; in situ hybridization; sodium bisulfite sequencing; tryptophan hydroxylase 2; TPH2; micropunch

Supplementary material for this article is available at www.BioTechniques.com/article/113912

Dissection of discrete brain regions for molecular analysis is complicated by trade-offs between accuracy, flexibility, and costs. We developed a flexible and cost-effective method, in situ hybridization (ISH) guided freeze-matrix assisted punches (IFAP), for extracting nanogram quantities of DNA from slide-mounted sections as thin as $12 \mu \mathrm{m}$. Using ISH to localize regions of interest, tissue is targeted by applying a small bead of M-1 embedding matrix onto cryosections, snap-freezing, and collecting the beads for nucleic acid purification. The method quantitatively recovers RNA and DNA usable for PCR and DNA methylation analysis.

DNA methylation is a mechanism by which long-term changes in gene expression are effected. Robust changes to the pattern of DNA methylation in homogeneous macroscopic tissues (e.g., carcinomas or cultured cells) are well established $(1,2)$. It has been posited that experience-dependent DNA methylation also occurs in healthy normal brain tissue, but data has remained limited and controversial (3). Issues pertaining to anatomical specificity, sample recovery, or high cost have limited progress.

The microdissection of tissue sections is tedious, requires thick sections, and is limited by punch availability. Recovery of tissue from fresh-frozen thin sections, suitable for anatomical studies, is all but impossible. We have developed a technique, based on the principle of in situ hybridization (ISH)-guided laser capture microdissection (LCM; 4), for punching portions of tissue from slide-mounted frozen thin sections. By applying a small bead of liquid onto cryosections and snap-freezing, underlying tissue is lifted when beads are pulled away, and DNA is extracted from bead-tissue complexes using conventional high-salt DNA extraction techniques. This method, ISH-guided freeze-matrix assisted punches (IFAP), facilitates rapid and efficient recovery of discrete regions under conditions suitable for DNA methylation analysis. IFAP utilizes commonly available histological resources to maintain low cost and allows for parallel analyses from source tissue.

The IFAP method synergizes well with standard histological workflows. Tissue processing is streamlined, with sections $(\geq 12 \mu \mathrm{m})$ mounted on $n$ slide sets (sections $1,1+\mathrm{n}, 1+2 \mathrm{n}$...on set 1 ; sections $2,2+n, 2+2 n$...on set 2 ; etc., up to sections $n, 2 n, 3 n$...on set $n)$. Each set represents a staggered survey through the region of interest. An ISH is performed on one set to localize the target area and is used to guide
IFAP dissection on an adjacent set. IFAP in principle works similar to LCM, but instead uses low temperatures to bond tissue with pipet-applied matrix beads. Although not comparable in spatial specificity to LCM, IFAP is low-cost, rapid, and useful in millimeter scale dissections that do not require single-cell resolution.

The default approach to isolating nuclei from surrounding tissue is the micropunch (MP), which utilizes a hollowed sharpened cylinder to punch tissue (5). However, IFAP offers several advantages: (i) flexibility in sample condition and (ii) workflow efficiency for parallel analyses. In our experience, MP is typically more effective on fresh tissues and requires thicker sections $(\geq 60 \mu \mathrm{m})$ if frozen and slide-mounted. Additional difficulty occurs if sections are mounted on charged or treated slides, which are commonly used for histochemistry to improve sample retention. A theoretical workaround is to alternate thick and thin slices along with slide coatings, but in practice that is cumbersome and complicated. IFAP offers a slice resolution of at least $12 \mu \mathrm{m}$ and works with previously stored slide-mounted sections, including charged slides. Thus, it introduces nothing new to the workflow and can be readily applied in a range of conditions. In this study, we detail the use of IFAP in excision of brain nuclei and evaluate sample integrity for use in assessing DNA methylation. We use tryptophan hydroxylase 2 (TPH2), a predominantly raphe-restricted gene, as our test gene.

For IFAP, three brains from postnatal day 60 c57/bl6 male mice were sectioned at $12 \mu \mathrm{m}$ and mounted on Superfrost slides (Thermo Fisher Scientific, Waltham, MA, USA). A fourth mouse brain, for MP comparisons, was sectioned by alternating between 12- and 100- $\mu \mathrm{m}$ slices, with the $12-\mu \mathrm{m}$ sections used for ISH-guidance and the $100-\mu \mathrm{m}$ sections reserved for MP. This alternation of section depth is necessary because MP is impractical on thinly sliced, frozen slide-mounted sections. Slides were stored at $-80^{\circ} \mathrm{C}$, then reequilibrated to $-20^{\circ} \mathrm{C}$ prior to dissection. All supplies were preequilibrated to $-20^{\circ} \mathrm{C}$ to prevent beads from reliquefying. For liquid application, TipOne ultra-low retention pipet tips (USA Scientific, Ocala, FL, USA) were used to minimize volume variance.

Initially, tests were run to characterize the suitability of different liquids-TE (10 $\mathrm{mM}$ Tris- $\mathrm{HCl}, 0.1 \mathrm{mM}$ EDTA, $\mathrm{pH} 8.0$ ), water, or M-1 embedding matrix (Shandon; Thermo Fisher Scientific) - for binding and lifting tissue. The rationale for testing M-1, a water-soluble embedding matrix conventionally used to support sectioning of frozen tissue, was that its viscosity would minimize 
A

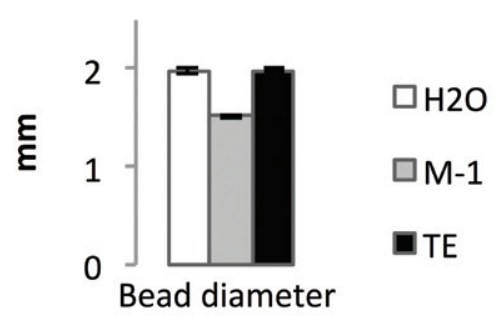

\section{$\mathbf{F}$}

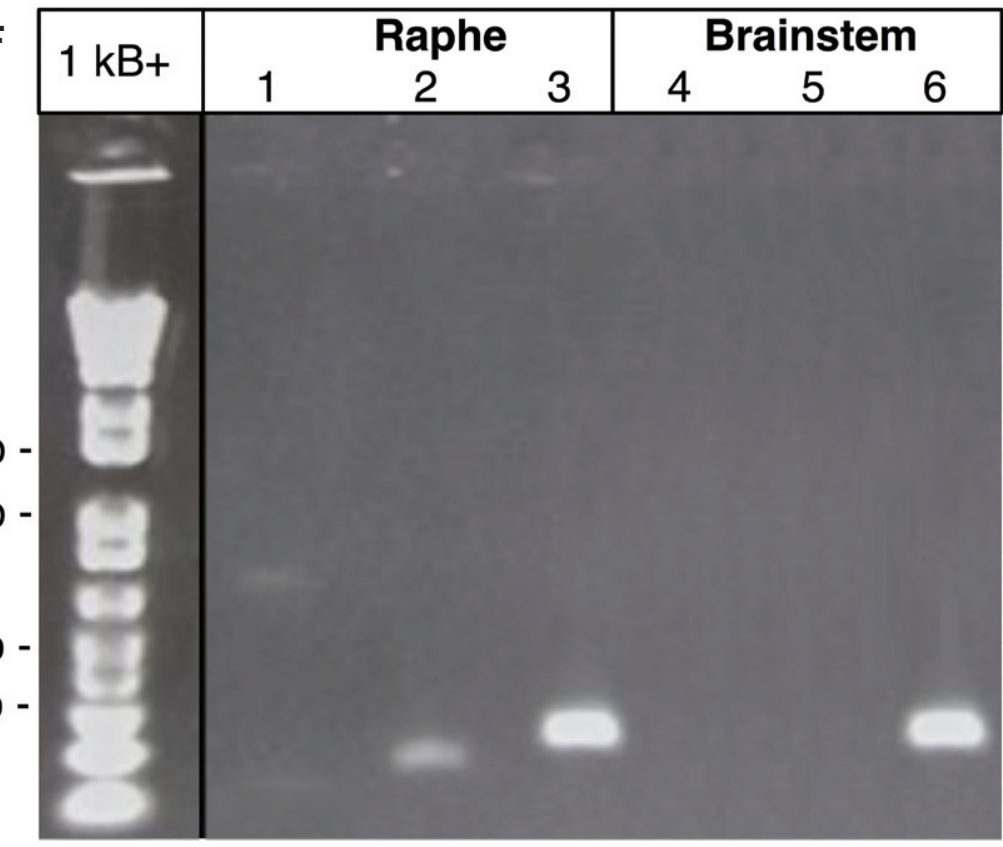

D

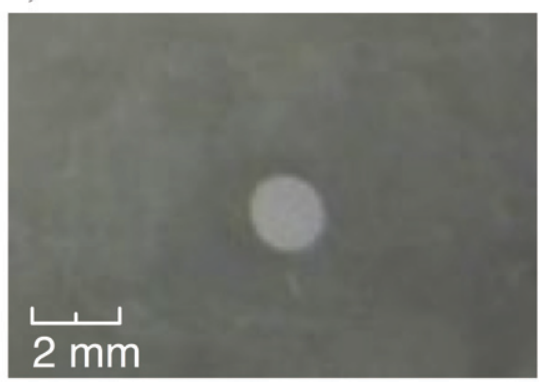

E

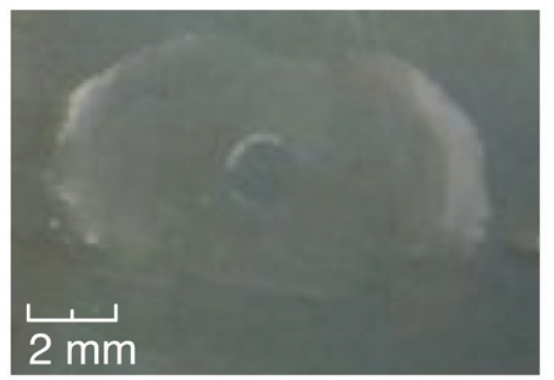

Figure 1. IFAP extraction of target nuclei and target specificity. (A) Mean \pm SEM of the average bead diameters. (B) Mean \pm SEM of standard deviations of six diameter measurements per bead. (C) TPH2 ISH delineates the region of interest. (D) Beads are snap-frozen over the region of interest. (E) Bead removal lifts tissue from the slide. (F) Amplification of target cDNA from reverse-transcribed extracted RNA, run on a 1\% TBE agarose gel. Raphe tissue was isolated using IFAP M-1. Brainstem tissue corresponds to an adjacent whole section. Lanes 1 and 4, LMX1b; lanes 2 and 5, TPH2; lanes 3 and 6, HPRT.

variability in bead diameter. Per liquid, beads were tested for $(i)$ dispersal and uniformity and (ii) structural integrity during removal. Diameters were measured (ImageJ) every $30^{\circ}$ across each bead. For $0.5 \mu \mathrm{L}$, the average bead diameter for M-1 was $\sim 1.5 \mathrm{~mm}$, whereas for $\mathrm{TE}$ and water it was $2 \mathrm{~mm}$ (Figure 1A). Moreover, bead diameter variance was less for M-1 compared to TE or water beads (Figure 1B), indicating better uniformity in bead dimensions. Qualitatively, water and TEbeads were more prone to fracture during removal from slides, negatively affecting recovery. For these reasons, M-1 was considered optimal for IFAP dissections.

To test IFAP specificity, the dorsal raphe, a region rich in serotonergic (5-HT) neurons, was targeted for extraction from surrounding brainstem tissue. ISH histochemistry for TPH2 (Figure 1C) guided IFAP dissection on adjacent sections (Figure 1D). The ISH was used to identify (i) raphe-containing sections and (ii) localize the tissue areas corresponding to the highest TPH 2 optical density within a $1.5 \mathrm{~mm}$ diameter circular area. For the full ISH protocol, see Reference 6. Template for sense and antisense probes were generated from cDNA [expressed sequence tag (EST): BG084420]. Using ISH as a reference, each slide was briefly brought to room temperature, and a $0.5-\mu \mathrm{L}$ aliquot of M-1 embedding matrix applied to each section on the slide. After application, slides were quickly placed onto dry ice to snap-freeze the bead (Figure 1D). For punch removal, slides were transferred to the cryostat, and the blunt edge of a razor blade was used to dislodge each bead from the slide, simultaneously lifting tissue (Figure $1 \mathrm{E})$. A total of 10 beads per sample was used. After all beads were collected in a chilled 1.5-mL tube, the tube was briefly centrifuged for $5-10 s$ at $\sim 4000 \times g$.

To verify IFAP accuracy, RNA was purified (TRIzoL; Invitrogen, Carlsbad, CA, USA), resuspended $(20 \mu \mathrm{L})$, reversetranscribed $(10 \mu \mathrm{L})$ using random 8-mers (SuperScript II; Invitrogen), and cDNA amplification from raphe-specific genes, TPH 2 and LMX1B, compared against RNA from an intact brainstem section from an adjacent slide. For cDNA amplification, the following exon-spanning primers were used: LMX1B: $\quad 5^{\prime}$-CTGCTGTGCAAGGGTGACTA-3'/5'-AAACCAGACCTGGACCACAC-3', TPH2: 5'-TGTCCTTGGATTCTGCTGTG$3^{\prime} / 5^{\prime}$ - GCCCACCA ACT TCATTCTTC-3', and HPRT: 5'-CAGTACAGCCCCAA A A TGGT-3'/ $5^{\prime}$ GCGCTCATCTTAGGCTTTGT-3'. As expected, because of the higher concentration of raphe tissue extracted using IFAP, IFAP samples successfully amplified TPH2 and LMX1B, whereas RNA from the brainstem section only amplified the ubiquitously expressed HPRT (Figure 1F). This confirms specificity for targeting nuclei and regionally restricted genes, as well as integrity of RNA by this method. Amplicons for TPH2 and LMX1B were TOPO cloned (Invitrogen) and sequence-verified. 
A CpG position:

31

35

Original: $\quad$ C T C T T CA G C C CA G G T T C T G G A A G C G C C C C G A G C

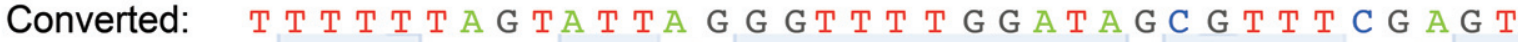

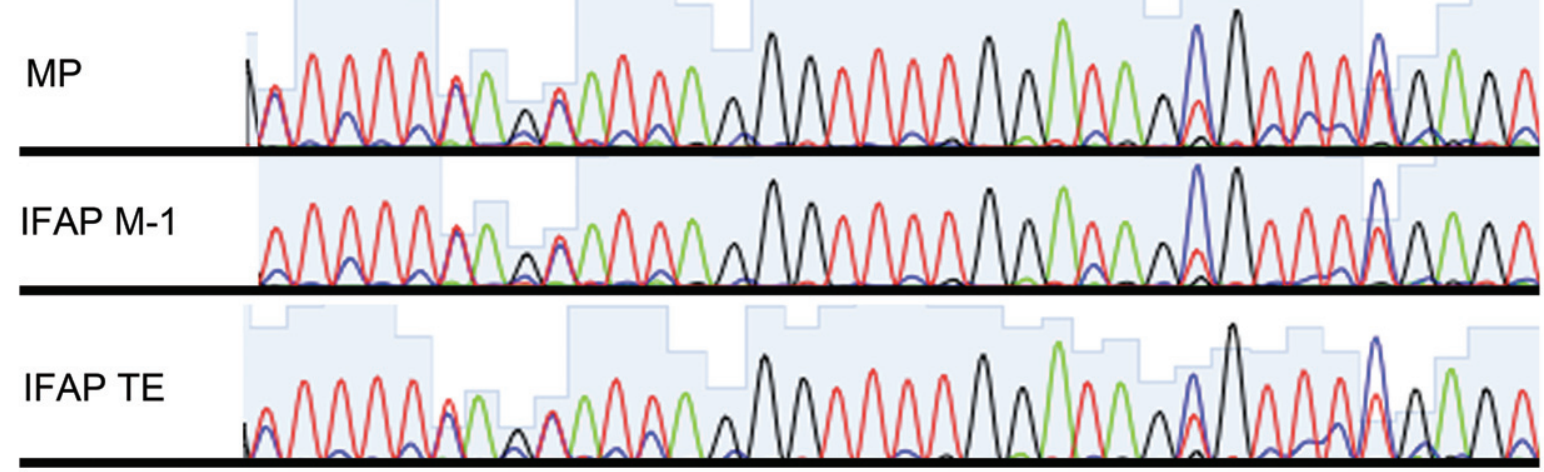

B

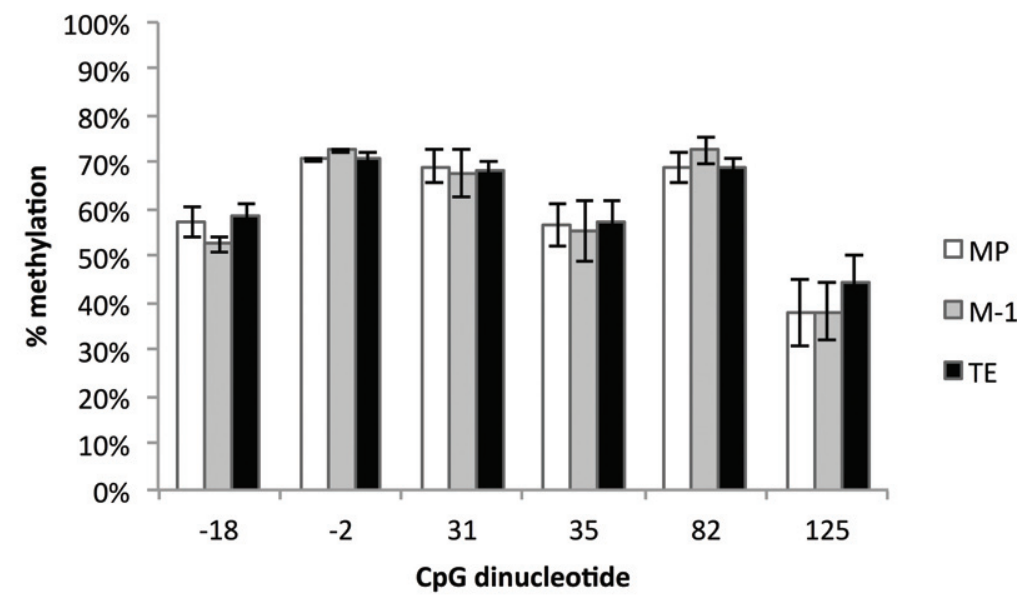

Figure 2. Analysis of DNA methylation using MP, IFAP M-1, or IFAP TE extracted samples at the TPH2 promoter and $5^{\prime}$ UTR. (A) Sequence chromatograms of sodium bsulfite-converted amplicons. Original and expected bisulfite-converted sequences are notated, and CpG positions marked relative to putative transcription start site at the promoter and 5' UTR junction. Bisulfite conversion of cytosine to thymine is comparable between MP and IFAP. (B) Methylation of $\mathrm{CpG}$ dinucleotides is indistinguishable between MP and IFAP.

For genomic DNA (gDNA) extraction, 10 beads per sample were treated to a standard high-salt TNES DNA extraction protocol (7) and resuspended in $25 \mu \mathrm{L}$ TE (10 mM Tris, $0.1 \mathrm{mM}$ EDTA). For TE beads, $1 \mu \mathrm{L} 10 \mathrm{mg} / \mathrm{mL}$ glycogen was added as a coprecipitant prior to precipitation, whereas M-1 contained an inherent coprecipitant (likely polyethylene glycol) and did not require glycogen.
As a result, DNA pellets were also more gelatinous and required additional time $(5 \mathrm{~min})$ and heat $\left(50^{\circ} \mathrm{C}\right)$ for resuspension. Although in our downstream tests, M-1's unknown proprietary ingredients had no adverse effect on bisulfite conversion or sequencing, Nanodrop (Thermo Fisher Scientific) readings revealed elevated peaks at 220-230 nm, which complicated accurate gDNA quantitation. This neces-

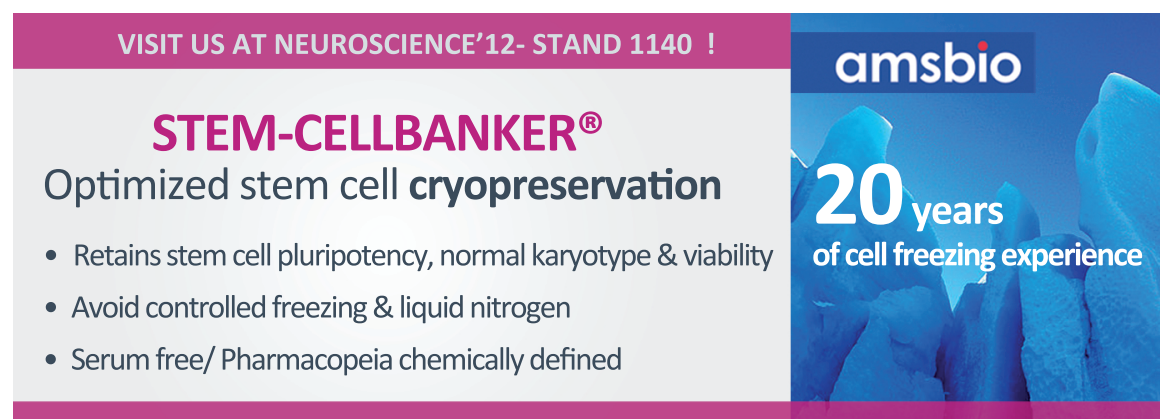

> FIND OUT MORE www.amsbio.com | info@amsbio.com sitated alternative quantitation by SYBR green fluorescence. Each gDNA sample was diluted 1:100 in TE, pH $7.5(10 \mathrm{mM}$ Tris, $0.1 \mathrm{mM}$ EDTA), and mixed with an equal volume of a 1:1000 dilution of SYBR green I (Invitrogen) in TE, pH 7.5, for a final volume of $200 \mu \mathrm{L}$. Fluorescence was compared to a DNA standard curve with $\mathrm{M}-1$ added $(0.5 \mu \mathrm{L}$ per bead in sample) to extrapolate yield. Fluorescence quantitation of gDNA from 10 beads returned $~ 400$ ng gDNA. For comparison, commercial kits typically return $\sim 1-3 \mu \mathrm{g}$ gDNA $/ \mathrm{mg}$ brain tissue. Based on $0.5 \mu \mathrm{L}$ IFAP beads generating $\sim 1.5 \mathrm{~mm}$ diameter punches of $12 \mu \mathrm{m}$ thickness, 10 punches would have an estimated mass of $\sim 212 \mu \mathrm{g}$ (assuming density of 1), returning 212-646 ng gDNA. Observed yield (400 ng) is well within expectations.

To test usability of IFAP-extracted samples for DNA methylation analysis, we assessed the sequence quality of gDNA obtained using IFAP (M-1 or TE) from slide-mounted thin sections 
TAKE ACTION AGAINST CONTAMINATION WITH $\mathrm{QMI}^{\circledR}$ SAFE-SEPTUM

\section{Sample, Inoculate Or Add Nutrients}

To Your Bioreactor Aseptically !

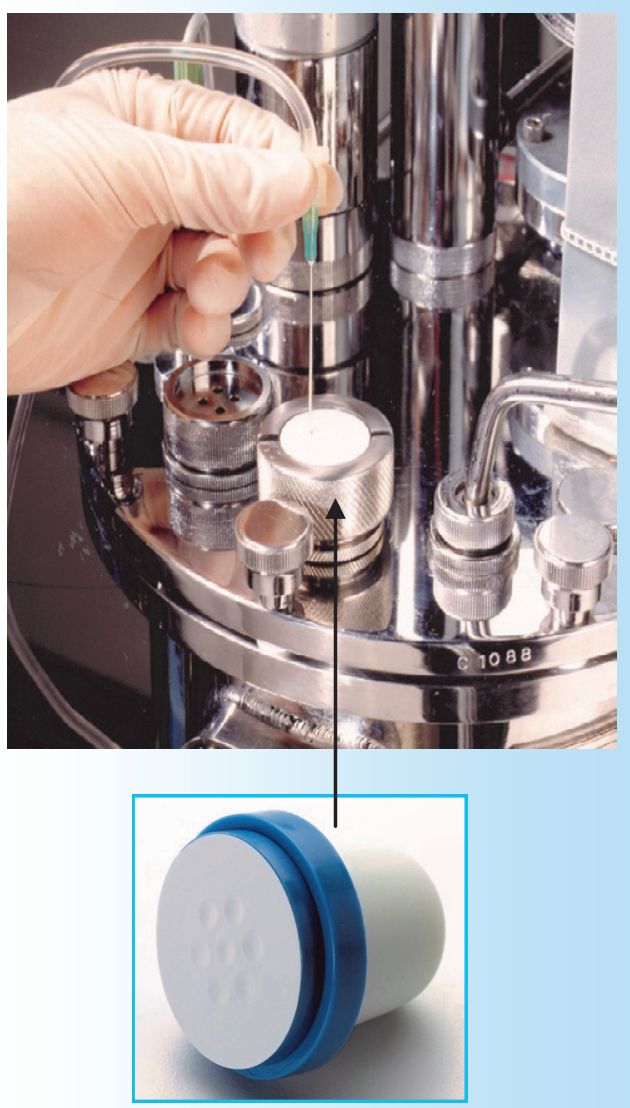

The QMI Safe-Septum is:

- Aseptic

- Pressure \& Temperature Safe

- Pre-Sterilized

- Easy To Retrofit

- Validated

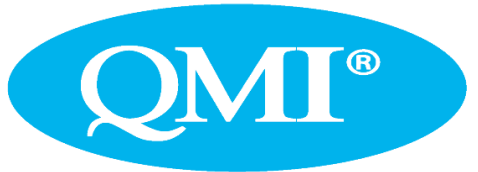

651-501-2337

Email: info@qmisystems.com

www.qmisystems.com
(10 beads) against tissue obtained using a $1.0 \mathrm{~mm}$ diameter MP (VWR International, Radnor, PA, USA) on thick sections and replicated across three independent samples. gDNA was converted with sodium bisulfite (EpiTect; Qiagen, Valencia, CA, USA), and DNA methylation was assessed at the TPH 2 promoter and $5^{\prime}$ untranslated region (UTR) using a nested PCR approach, with inner nested primers fused to an M13 adaptor to improve sequencing. Outer nested primers were: 5'-TAGAYGTGTAATTTGATTGTGGTTATTAGT-3' and 5'-TCCCAACAACT-CRCCCAACTAC-3'. Inner nested primers were: $5^{\prime}$-M13fwd-TGATTGTGGTTATTAGTAATT-AGAAATGAGTT-3' and 5'-M13rev-AATCCAAAAACAACCCTCTCC-3'. Amplicons were column-purified (QIAquick; Qiagen) and sequenced bidirectionally (Model 3730 XL; Applied Biosystems, Foster City, CA, USA). Percent methylation was estimated by comparing cytosine:thymine peak ratios in $\mathrm{CpG}$ dinucleotides $[\%$ methylation $=(\mathrm{C}) /$ $(\mathrm{C}+\mathrm{T})]$. Sequence chromatograms of MP and IFAP M-1 or TE indicated comparable conversion of unprotected cytosines (Figure 2A). Furthermore, no difference in CpG dinucleotide methylation was observed, indicating minimal IFAP interference with bisulfite conversion (Figure 2B).

Because there is, to our knowledge, no publication of brain nuclei specific differences in methylation pattern, we are unable to definitively ascertain IFAP's sensitivity for detecting methylation differences. Although this method minimizes inclusion of non-nuclei tissue by reducing section thickness requirements, it does remain limited by cell heterogeneity (i.e., neuronal versus glial) in the target nuclei and is unable to discriminate cell-type restricted changes in DNA methylation. Notwithstanding this limitation, the successful extraction of RNA, conversion, and amplification of $\mathrm{TPH} 2$ and $\mathrm{LMX} 1 \mathrm{~b} c \mathrm{CDNA}$ suggests a level of sensitivity comparable to MP, while offering increased targeting accuracy and streamlined processing when using conventional slide-mounted frozen tissue sections.

In conclusion, IFAP is a flexible, low-cost, and simple method for dissecting nuclei from thin slide-mounted sections. The method synergizes well with conventional histochemical techniques, allowing for parallel investigations with tissue derived from the same animal. The central advantages of IFAP over MP are $(i)$ seamless integration into the histochemical workflow, (ii) quantitative recovery from thin, slidemounted frozen sections, (iii) no need for specialized equipment, and (iv) targeting accuracy using ISH. We verified that gDNA and RNA extracted by this method can be used in DNA methylation analysis and cDNA synthesis, respectively.

\section{Acknowledgments}

We would like to thank Delia Vazquez, Juan Lopez, Robert Thompson, and Robert Denver for their input. This work was supported by a grant from the Pritzker Neuropsychiatric Disorders Research Consortium, which is supported by the Pritzker Neuropsychiatric Disorders Research Fund L.L.C. and a NARSAD Young Investigator Grant to P.D.P. (N008728).

\section{Competing interests}

The authors declare no competing interests.

\section{References}

1. Jones, P.A. and S.B. Baylin. 2002. The fundamental role of epigenetic events in cancer. Nat. Rev. Genet. 3:415-428.

2. Jaenisch, R. and A. Bird. 2003. Epigenetic regulation of gene expression: how the genome integrates intrinsic and environmental signals. Nat. Genet. 33:245-254.

3. Mill, J., T. Tang, Z. Kaminsky, T. Khare, S. Yazdanpanah, L. Bouchard, P. Jia, A. Assadzadeh, et al. 2008. Epigenomic profiling reveals DNA-methylation changes associated with major psychosis. Am. J. Hum. Genet. 82:696-711.

4. Bernard, R., I.A. Kerman, F. Meng, S.J. Evans, I. Amrein, E.G. Jones, W.E. Bunney, H. Akil, et al. 2009. Gene expression profiling of neurochemically defined regions of the human brain by in situ hybridization-guided laser capture microdissection. J. Neurosci. Methods 178:46-54.

5. Palkovits, M. and M.J. Brownstein. 1983. Microdissection of brain areas by the punch technique, p. 1-36. In A.C. Cuello (Ed.), Brain Microdissection Techniques. Wiley, New York.

6. Vazquez, D.M., C.R. Neal, Jr., P.D. Patel, N. Kaciroti, and J.F. Lopez. 2012. Regulation of corticoid and serotonin receptor brain system following early life exposure of glucocorticoids: long term implications for the neurobiology of mood. Psychoneuroendocrinology 37:421-437.

7. Maniatis, T., E.F. Fritsch, and J. Sambrook. 1983. Molecular Cloning: A Laboratory Manual, p. 545. CSH Laboratory Press, Cold Spring Harbor, NY.

Received 14 May 2012; accepted 6 July 2012.

Address correspondence to Lawrence S. Own, 109 Zina Pitcher Place, BSRB Room 5698, Ann Arbor, MI, USA.Email: aureias@umich.edu

To purchase reprints of this article, contact: biotechniques@fosterprinting.com 